\title{
Integration of Off-Track Sonic Boom Analysis in Conceptual Design of Supersonic Aircraft
}

\author{
Irian Ordaz* and $\mathrm{Wu} \mathrm{Li}^{\dagger}$ \\ NASA Langley Research Center, Hampton, VA 23681
}

\begin{abstract}
A highly desired capability for the conceptual design of aircraft is the ability to rapidly and accurately evaluate new concepts to avoid adverse trade decisions that may hinder the development process in the later stages of design. Evaluating the robustness of new low-boom concepts is important for the conceptual design of supersonic aircraft. Here, robustness means that the aircraft configuration has a low-boom ground signature at both under- and off-track locations. An integrated process for off-track boom analysis is developed to facilitate the design of robust low-boom supersonic aircraft. The integrated off-track analysis can also be used to study the sonic boom impact and to plan future flight trajectories where flight conditions and ground elevation might have a significant effect on ground signatures. The key enabler for off-track sonic boom analysis is accurate computational fluid dynamics (CFD) solutions for off-body pressure distributions. To ensure the numerical accuracy of the off-body pressure distributions, a mesh study is performed with Cart3D to determine the mesh requirements for offbody CFD analysis and comparisons are made between the Cart3D and USM3D results. The variations in ground signatures that result from changes in the initial location of the near-field waveform are also examined. Finally, a complete under- and off-track sonic boom analysis is presented for two distinct supersonic concepts to demonstrate the capability of the integrated analysis process.
\end{abstract}

\section{Nomenclature}

Acronyms

$\begin{array}{ll}\text { CFD } & =\text { computational fluid dynamics } \\ \text { OFF } & =\text { object file format } \\ \text { STL } & =\text { stereo lithography } \\ \text { TRI } & =\text { Cart3D surface triangulation } \\ \text { VSP } & =\text { Vehicle Sketch Pad } \\ \text { XML } & =\text { extensible markup language }\end{array}$

Symbols

$d p / p \quad=$ near-field waveform

$S_{\text {ref }} \quad=$ reference area

\section{Introduction}

$\mathrm{T}$ HIS paper focuses on the integration of off-track sonic boom analysis into the conceptual design of supersonic aircraft. Computational fluid dynamics (CFD) techniques for accurate off-body pressure prediction have advanced rapidly in the last several years. ${ }^{1-3}$ Relatively accurate off-body pressure distributions can now be obtained several body lengths below the aircraft, although the computational cost is substantially higher than for computing accurate surface pressures.

\footnotetext{
* Research Engineer, Aeronautics Systems Analysis Branch, Systems Analysis and Concepts Directorate.

${ }^{\dagger}$ Senior Research Engineer, Aeronautics Systems Analysis Branch, Systems Analysis and Concepts Directorate.
} 
The current analysis and design process $^{4,5}$ for supersonic concepts at NASA Langley Research Center uses an automated USM3D ${ }^{6,7}$ CFD analysis to conduct high-fidelity aerodynamics analysis. This automated mesh generation and CFD analysis requires several hours to obtain off-body pressures for a conceptual configuration. Preliminary results indicate that Cart3D ${ }^{8}$ requires relatively less time than USM3D to obtain adequate solutions for both surface and off-body pressures. Moreover, Cart3D is more flexible regarding its input geometry format. It can accept any triangulated surface of the input geometry or a collection of solid components that are defined in Plot3D format. USM3D relies on VGRID, ${ }^{9,10}$ which requires an accurate definition of the outer mold line of a watertight CFD geometry. Because defining the solid components of a configuration is easier than defining the corresponding watertight geometry, Cart3D is generally more suitable for CFD analysis during the conceptual design phase.

As a result, the integration of Cart3D into the current supersonic analysis and design process is desirable so that the analysis results of USM3D and Cart3D can be compared for the same configuration. The previous integration effort for Cart3D was based on the surface triangulation capability of Vehicle Sketch Pad $^{11}$ (VSP) to generate a coarse surface mesh for a configuration. ${ }^{12}$ Such a coarse surface mesh makes comparison with the USM3D results unrealistic because the surface mesh that is generated by VGRID is a higher quality. Thus, further improvements have been made to the automated Cart3D analysis for conceptual configurations to make the comparison study with USM3D possible. Moreover, a new and more sophisticated sonic boom propagation code sBOOM ${ }^{13}$ is used to propagate Cart3D offbody pressures and obtain off-track ground signatures. To facilitate a fast turnaround time for conceptual design, an automated analysis process that starts with a conceptual parametric geometry defined in VSP and ends with the generation of off-track ground signatures based on Cart3D analysis is implemented in ModelCenter. ${ }^{14}$ A study that uses this integrated boom analysis capability is performed with Cart3D to assess the sensitivity of ground signatures to both variations in grid resolution and the initial near-field waveform location for a low-boom supersonic concept.

\section{Automated Surface Mesh Generation}

The capability for automated volume mesh generation in Cart3D is a key enabler to its application in the conceptual design phase. Another key enabler for the automated CFD analysis of conceptual design geometries is a tool for automated surface meshing.

VSP is a conceptual geometry package that allows parameterization and manipulation of fuselages, wings, nacelles, pods, engines, and so on. It also includes easy-to-use CFD surface meshing ${ }^{15}$ capabilities that can be automated in batch mode. The process to obtain a surface triangulation of a conceptual geometry for Cart3D using VSP is described by Ordaz. ${ }^{16}$ The process starts with a VSP parametric geometry in extended markup language (XML) format and uses the built-in CFD surface meshing capability of VSP.

The Cart3D package requires a closed surface triangulation (TRI) as input to the Cartesian volume mesh generator. Included in the Cart3D package is a tool that allows the triangulation of componentbased surface point definitions in Plot3D and LaWGS. Additional tools are included for the conversion of surface triangulations in stereo lithography (STL) and other CAD-based formats (i.e., OFF) to the Cart3D input mesh format (i.e., TRI).

VSP can also be used to create a surface triangulation of a geometry in a component-based point definition format (i.e., Hermite or Plot3D). The component-based point definition format that is exported by VSP is called Hermite. The Hermite format is similar to Plot3D but contains more detailed component information, including the specification of lifting and nonlifting surfaces. This requires the reconstruction of a VSP model in XML format from the Hermite format, as described by Ordaz. ${ }^{16}$ Once the XML model is constructed, the process that is described by Ordaz $^{16}$ can be used to obtain the final surface triangulation. The benefit of this approach is the use of the VSP built-in and high-quality CFD meshing capability for non-VSP geometry models.

A tool called GEO2VSP ${ }^{16}$ was developed to allow for the geometrical reconstruction of a fully parametric VSP model from a geometry model that is defined in Hermite or Plot3D format. GEO2VSP can currently handle any number of fuselage, pod, wing, and nacelle-type components.

Bezier curve parameterization has been implemented for nonstandard and complex fuselage cross sections. This means that complex cross sections can be manipulated via the "Edit" menu in the fuselage property box within VSP. This provides the best curve fit with the minimum number of Bezier segments for smooth sections. The tool is capable of handling nonsmooth sections; however, the fit around discontinuities will not be exact, and the number of Bezier segments will significantly increase. Fuselage cross section discontinuities can be handled without difficulty if they occur at the $X Z$ symmetry plane (i.e., a keel). A sample reconstructed geometry that consists of distinct and challenging features is shown in Fig. 1(a). In this figure of the ring wing, the shaded surface denotes the original point data, and the 
wireframe denotes the reconstructed model.

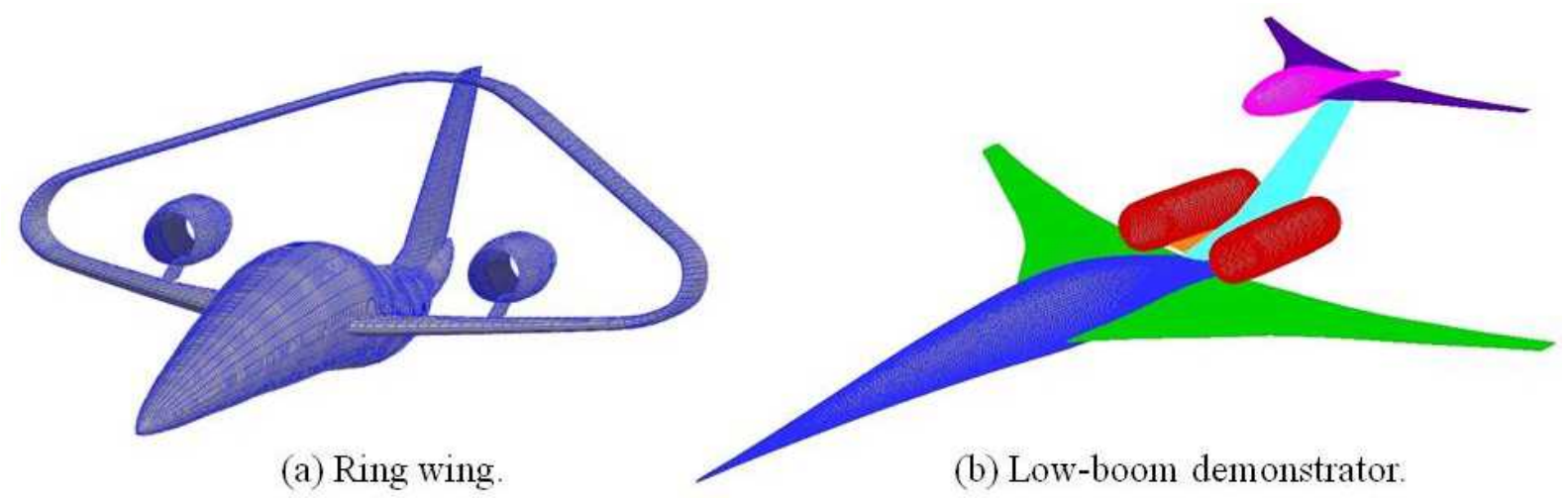

Figure 1. Supersonic concepts.

However, surface meshes that are generated by VSP are monolithic geometries, and individual components are not distinguishable. A monolithic surface mesh cannot be used for more complex analyses, such as excluding the nacelle interior surface in lift calculations or specifying boundary conditions on the nacelle exit area for engine simulations.

To obtain a component-based surface triangulation of a VSP configuration, one can use a point definition of each component of the VSP configuration in Hermite format. Figure 1(b) shows a componentbased surface mesh of a low-boom demonstrator ${ }^{17}$ in Hermite format. A component-based surface mesh from a conceptual configuration in Hermite format can be obtained by using (1) a code to generate solid components in Plot3D format from a VSP configuration in Hermite format and (2) the component-based surface meshing capability of Cart3D. This process is shown in Fig. 2, where a conceptual configuration in Hermite format is converted to a collection of solid components in Plot3D by using a computer code called HRM2GEO (see section IV of Geiselhart et al. ${ }^{18}$ ).

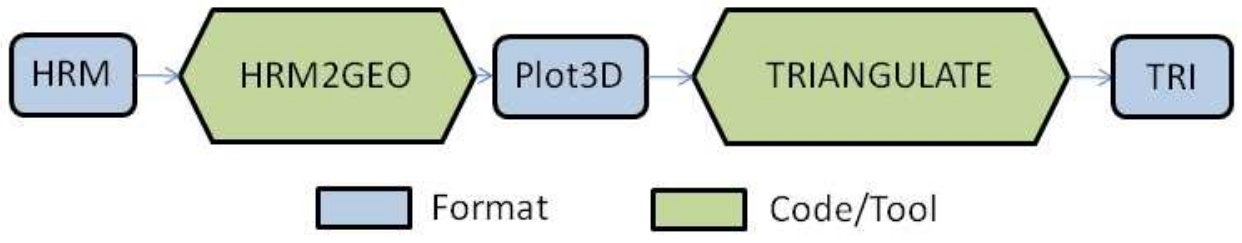

Figure 2. Process to convert conceptual configuration in Hermite format to component-based surface mesh.

Finally, note that Cart3D also can use an existing triangulated surface such as a VGRID surface mesh. A tool called VGRID2TRI has been integrated into the ModelCenter process to allow the conversion of a VGRID surface mesh to the Cart3D TRI format. This conversion tool was developed by Richard Campbell of NASA Langley Research Center. As a result, USM3D and Cart3D solutions based on the same surface triangulation can now be compared.

\section{Integrated Off-Track Sonic Boom Analysis}

This section introduces the analysis tools and modeling techniques that are used in the current formulation, including synthesis of analysis tools, aerodynamics analysis, and sonic boom propagation.

\section{A. Integration of Analysis Codes}

Once the surface meshing process has been automated for conceptual geometries, other analysis codes can be integrated into ModelCenter for automated off-track sonic boom analysis. The integrated analysis process is illustrated by the flowchart in Fig. 3 . 


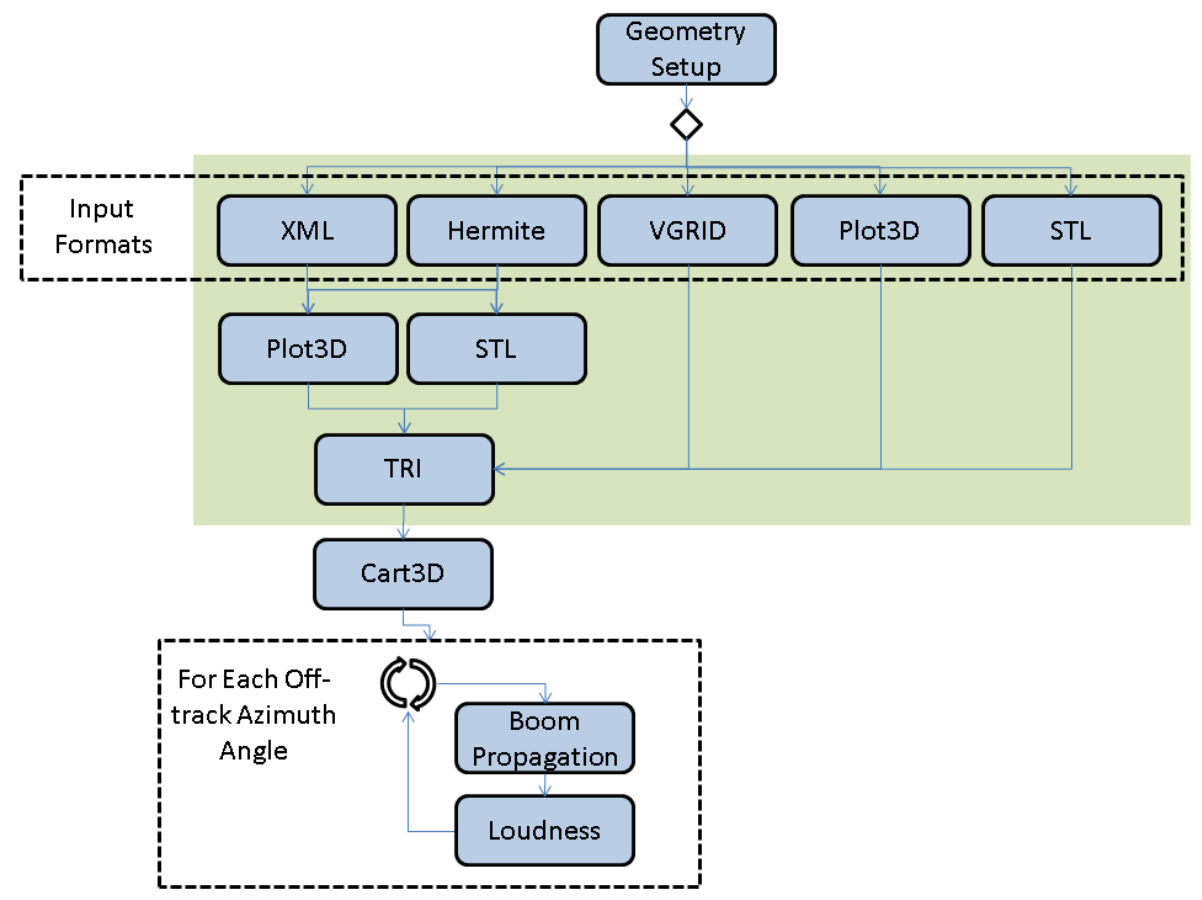

Figure 3. Integrated off-track sonic boom analysis process.

The new process flow modeling feature in ModelCenter simplifies the specification of process flow paths based on conditional logic, which in the past would have required more extensive programming to achieve. The built-in for-loop template simplifies the sequential computation and propagation of sonic boom signatures and loudness calculations at varying azimuth angles.

The automated surface mesh generation for various input geometry formats is implemented by using the logic nodes in the process flow. The various geometry paths in the model are activated depending on the type of input geometry format that is specified by the user (e.g., VSP XML, Hermite, STL, VGRID). ModelCenter wrappers have been developed for each of these geometry conversion tools.

A Cart3D wrapper has been developed to integrate Cart3D into ModelCenter. The wrapper provides the user with control over numerous inputs that range from the number of processors (shared or nonshared memory architecture of computer systems), flight conditions, refinement levels, lift matching, as well as the ability to extract line and point sensors, and planar and cylindrical cuts. In addition, the capability to perform automated adjoint and knowledge-based mesh adaptation has been added to allow the computation of more accurate off-body signatures for further propagation to the ground.

\section{B. CFD Analysis for Off-body Pressures}

Cart3D is a high-fidelity inviscid analysis CFD package that is geared toward conceptual and preliminary aerodynamics design. ${ }^{8}$ The package allows for quick and automated Cartesian-based volume mesh generation and CFD solutions for complex geometries. It includes numerous tools that facilitate the conversion and import of various geometry formats. In addition, it is highly scalable and can be run on multiprocessor systems.

For postprocessing, Cart3D can export plane cuts for flow parameters at arbitrary locations, as well as line data for pressures at off-body locations. Figure 4 shows a sample pressure contour on the symmetry plane that was obtained by using Cart3D for a low-boom demonstrator with flow-through nacelles at Mach 1.6 and an angle of attack of 0.49 deg. The volume mesh is rotated by Mach angle to align the shocks with the computational grid and consists of stacked refinement boxes to improve mesh efficiency. This process is used for all Cart3D off-body analyses in this paper and is automated for geometries in any of the five formats that are specified in Fig. 3. To obtain the off-track analysis results, a user need only select several input parameters, such as flight conditions, volume mesh refinement method and level, and the desired near-field distance for the computation of the off-body $d p / p$. 


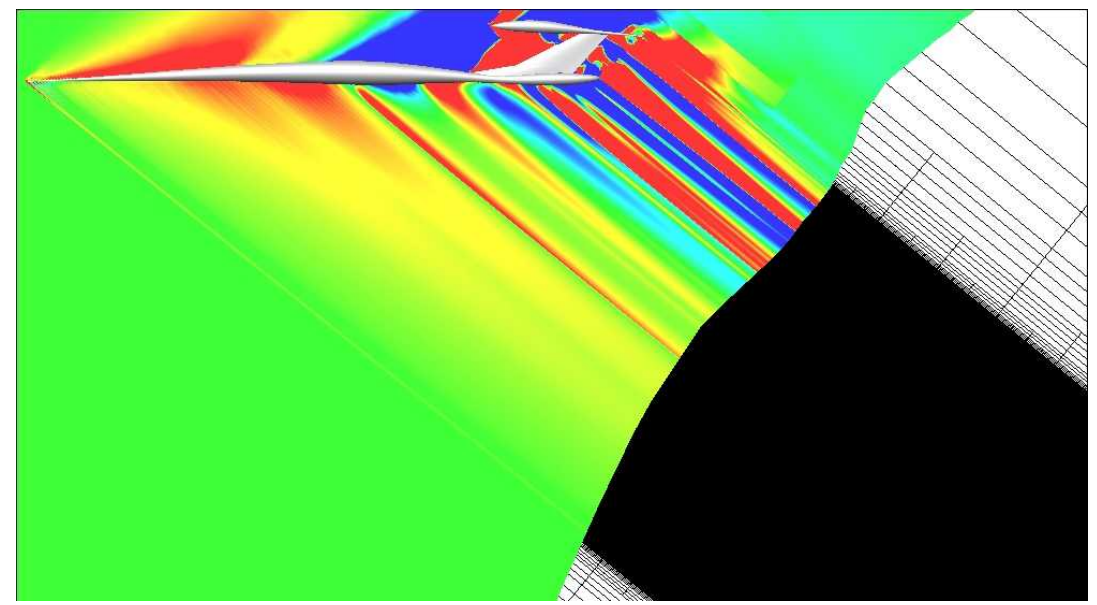

Figure 4. Cart3D symmetry plane pressure solution with stacked and rotated volume mesh.

\section{Sonic Boom Propagation}

The sonic boom analysis is conducted by using Cart3D knowledge-based mesh adaptation to generate off-body pressures at a constant $z$-location. This near-field waveform, which is shown in Fig. 5(a), is then propagated by using a new NASA propagation code called $\mathrm{sBOOM}^{13}$ to obtain the ground signatures that are shown in Fig. 5(b). sBOOM takes into account nonlinearity, molecular relaxation, and thermoviscous absorption and is capable of off-track propagation given a near-field waveform. A comparison of the SBOOM and the PCBOOM results is provided in Fig. 5(b); this comparison demonstrates good agreement between the two codes in cases where absorption and molecular relaxation are not used by sBOOM. The figure also shows the significant effects on the ground signature when absorption and molecular relaxation are enabled in sBOOM. The finite rise time of the ground signature when absorption and molecular relaxation are used by sBOOM leads to more accurate loudness calculations.

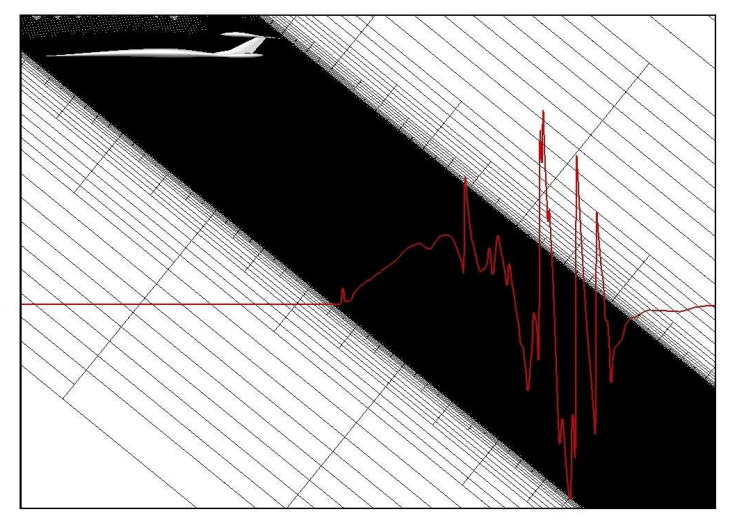

(a) Cart3D near-field $d p / p$ for propagation.

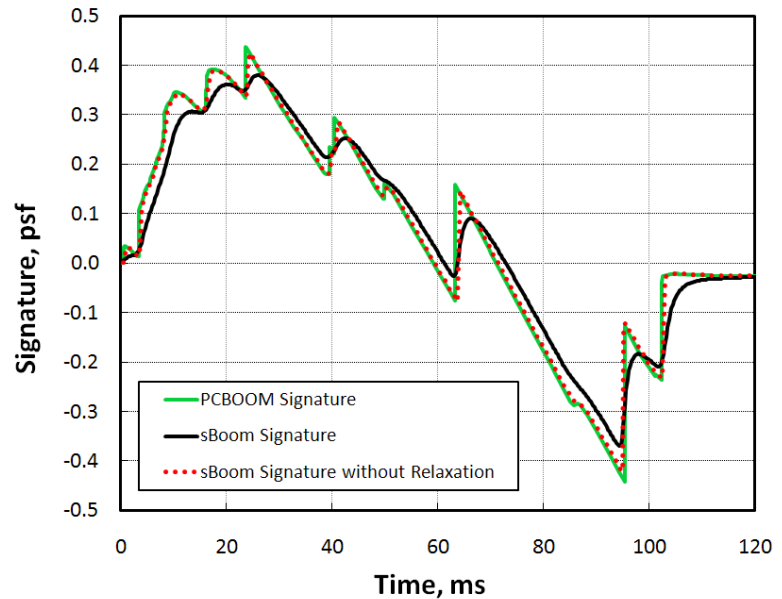

(b) Ground signature comparison of sBOOM and PCBOOM.

Figure 5. Sonic boom analysis process.

\section{Sensitivity of Solution Due to Differences in Mesh and Methods}

In this section, several studies are conducted to determine the sensitivity of the CFD solution with respect to the mesh refinement, the consistency of the off-body Cart3D solution with other established CFD codes (USM3D), and the sensitivity of the ground signature to the location of the starting nearfield waveform. The analysis results that are presented in this section were generated for the low-boom demonstrator at a Mach number of 1.6, an altitude of 47,500 ft, and an angle of attack of $0.49 \mathrm{deg}$.

The objective of the first study was to determine the sensitivity of the ground signature to the location of the near-field waveform $(d p / p)$ to be propagated. The $d p / p$ results at one, three, four, and five body 
lengths are shown in Fig. 6. The ground signatures for each $d p / p$ were propagated with sBOOM. The corresponding ground signatures are shown in Fig. 7. The study shows that for locations of four body lengths or greater the three-dimensional effects were successfully captured. Notice that the $d p / p$ at one body length still maintains an acceptable degree of accuracy, with loudness levels within 1.7 percent of the other results.

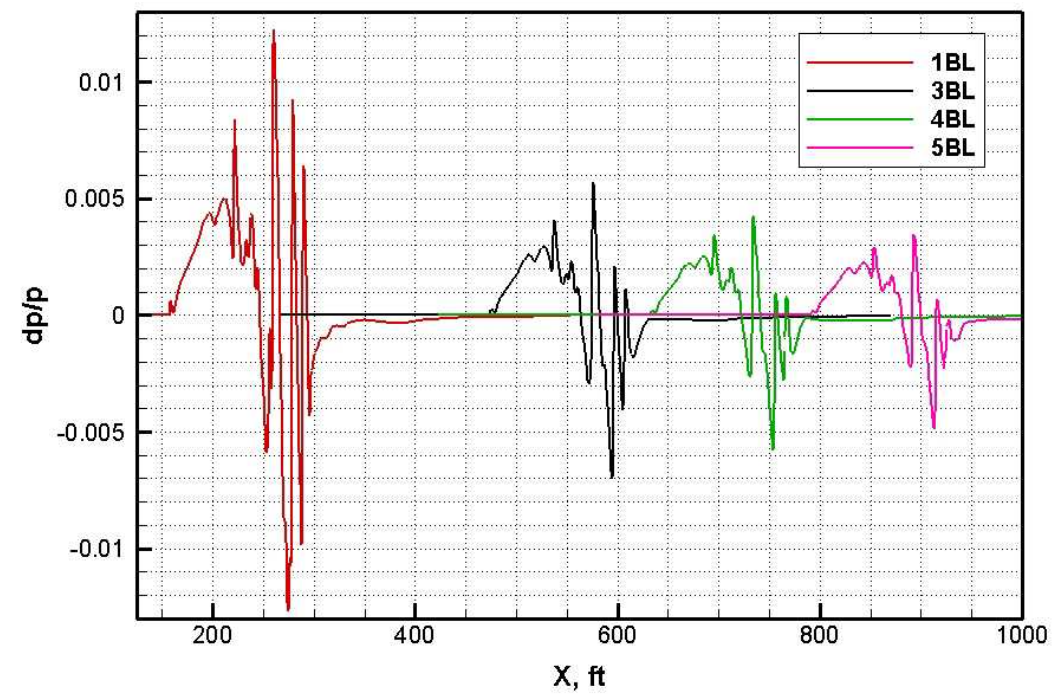

Figure 6. Cart3D $d p / p$ for low-boom demonstrator at several body lengths.

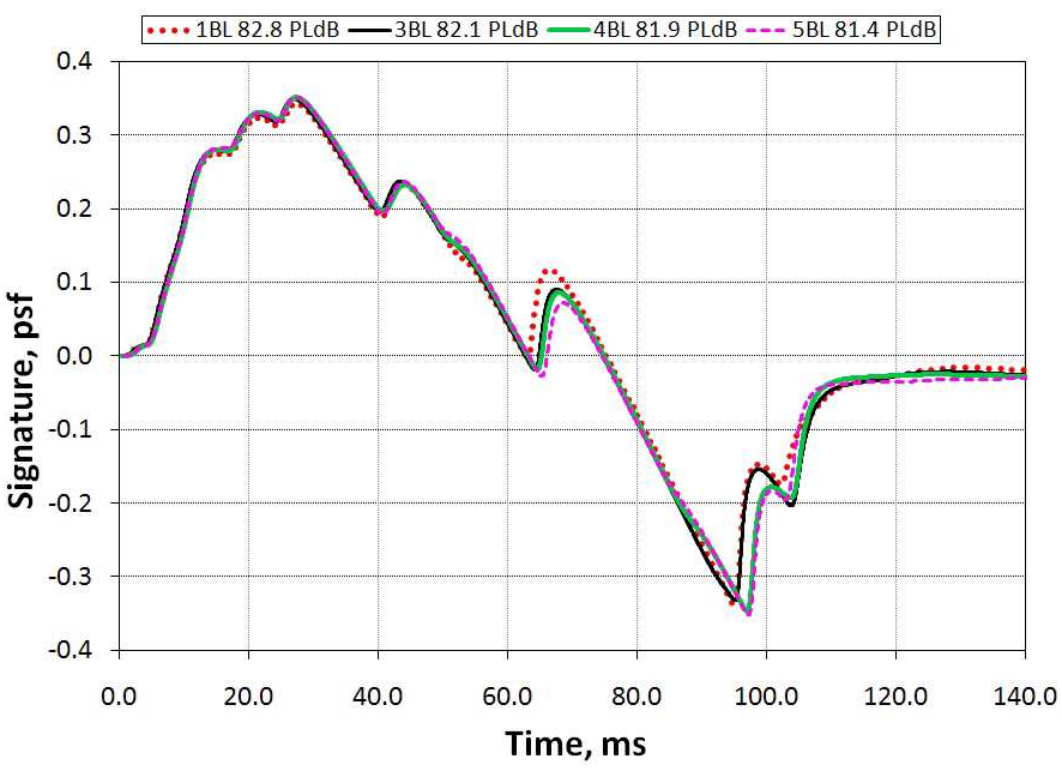

Figure 7. Sensitivity of ground signature to initial $d p / p$ location.

Subsequently, a mesh refinement was conducted to determine the sensitivity of the $d p / p$ to the CFD mesh refinement. The Cart3D $d p / p$ solutions for the low-boom demonstrator at three body lengths are given in Fig. 8 for three refinement levels. The number of points that are given in this plot denotes the number of points that describe the $d p / p$ over the computed domain. As suspected, increased refinement contributed to better resolution of the peaks and valleys in the $d p / p$. The $d p / p$ solutions were then propagated by using sBOOM, and the corresponding signatures are shown in Fig. 9. For this lowboom configuration, the maximum variation in loudness for the three signatures was within 1 percent. This result suggests that although small variations occur in the signature and loudness as a result of different refinement levels, coarser solutions still produce acceptable accuracy levels at a significantly 
lower computational cost.

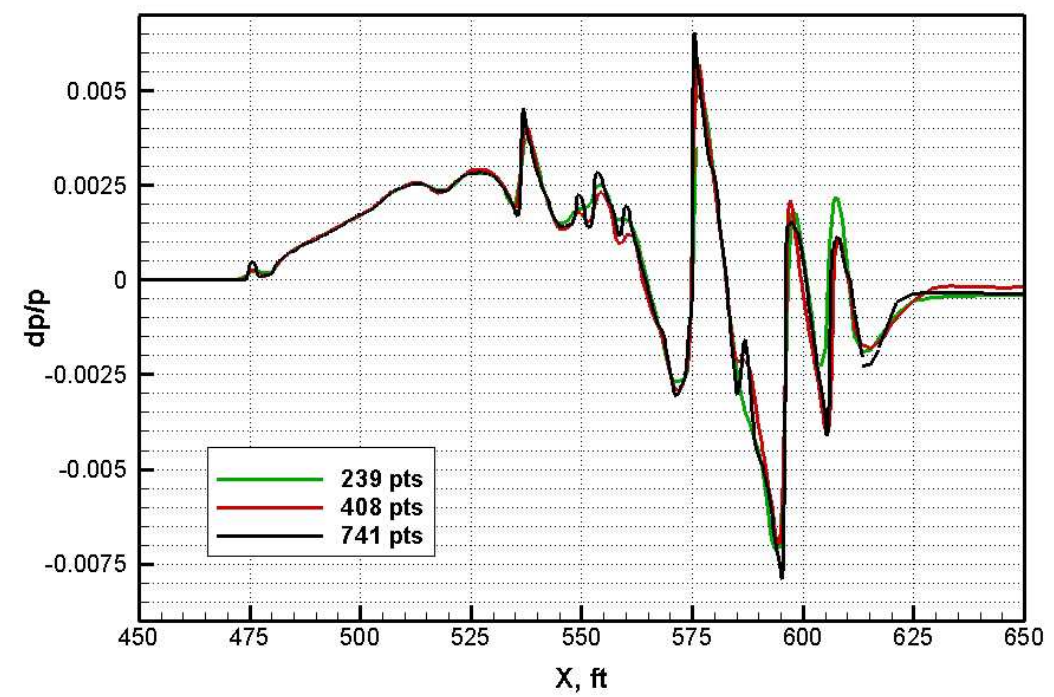

Figure 8. Cart3D $d p / p$ for low-boom demonstrator at three body lengths for various levels of volume mesh refinement.

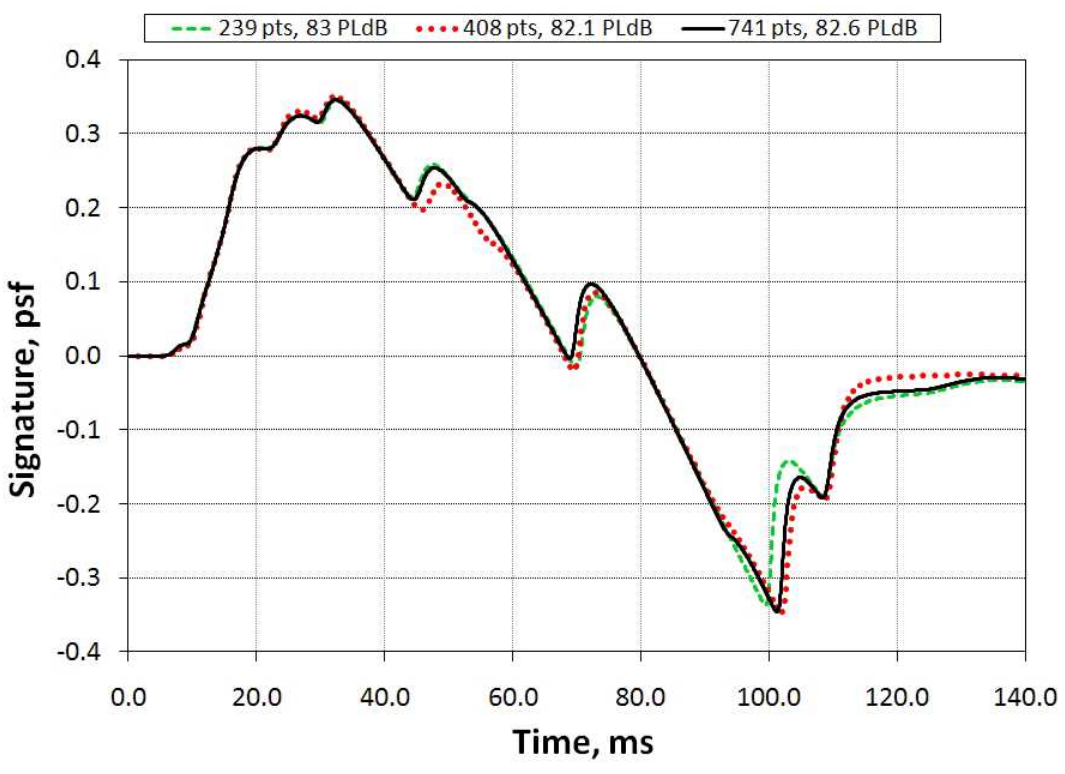

Figure 9. Sensitivity of ground signature to volume mesh refinement.

A near-field CFD solution and ground signature comparison study was also performed with Cart3D and USM3D. The Cart3D solution was obtained by using a fixed, stacked, and rotated mesh refinement to resolve the shocks and expansions in the near-field. USM3D solutions were obtained by using a stretchedgrid method to decrease the number of cells that were required for accurate off-body solutions. ${ }^{19}$ The near-field $d p / p$ results at three body lengths in the under-track location are shown in Fig. 10. The results show good agreement between the two codes despite the use of different analysis methods. 


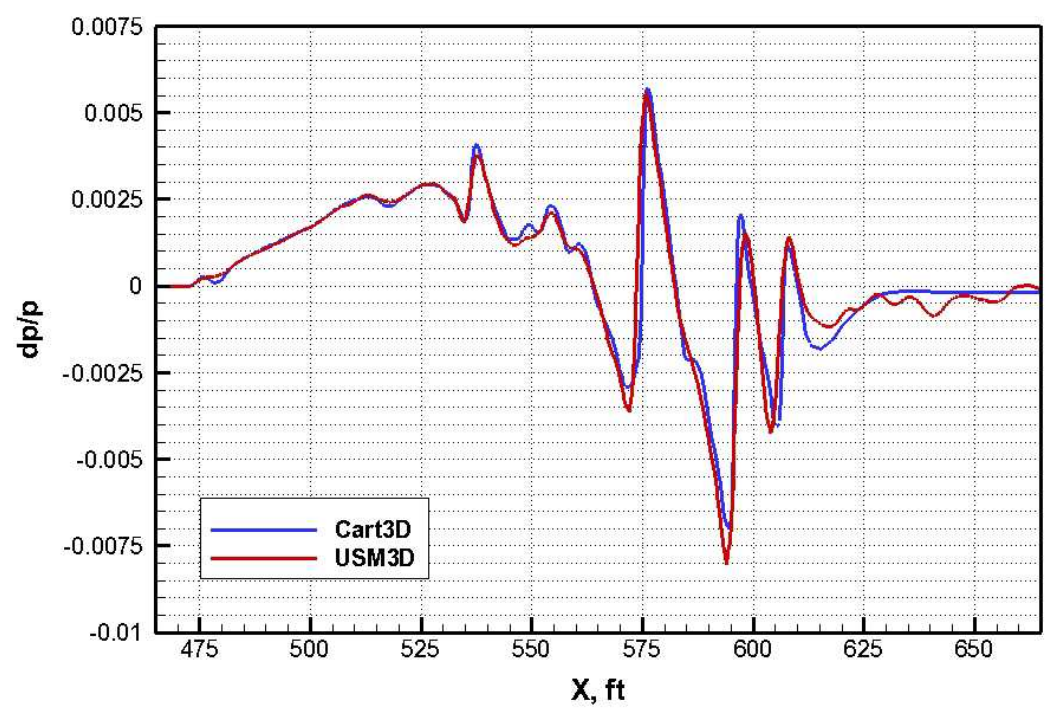

Figure 10. Comparison of Cart3D and USM3D off-body $d p / p$ results at three body lengths for low-boom demonstrator.

For the analysis results that are presented in the next section, the CFD analysis is performed with a level of refinement of approximately $400 d p / p$ points. The ground signature is computed from the near-field waveform at one body length to allow greater spanwise refinement in the off-track direction.

\section{Analysis Results}

In this section, the off-track analysis capability for conceptual design in ModelCenter is demonstrated. The full aerodynamic and sonic boom analyses for two different supersonic concepts are provided to demonstrate the robustness and flexibility of the process. The geometric information for the two concepts is provided in Table 1. (See Fig. 1 for the overall shapes of these two concepts.)

Table 1. Supersonic Concepts

\begin{tabular}{lcc}
\hline Concept & Length $(\mathrm{ft})$ & $S_{\text {ref }}\left(f t^{2}\right)$ \\
\hline \hline Ring wing & 170 & 931 \\
Low-boom demonstrator & 127 & 688 \\
\hline
\end{tabular}

The flow fields for the ring wing and the low-boom demonstrator were computed by Cart3D at angles of attack of 0.3 and 0.49 , respectively. The flight conditions for both vehicles were Mach 1.6 and an altitude of 47,500 ft. The objective of these results was not to draw direct comparisons between the boom characteristics of the two configurations because the ring wing concept was not carefully designed for low-boom characteristics. Instead, these configurations have distinct characteristics to demonstrate the capabilities of the automated analysis process.

The ground signatures, which were computed by using sBOOM for the under-track location (i.e., 0 deg) and azimuth angles of 38.2, 40.6, and $42.9 \mathrm{deg}$ for both concepts, are shown in Figs. 11 and 12. However, a more complete picture of the sonic boom signatures was obtained by plotting the sonic boom carpets, as shown in Fig. 13. The carpets depict the ground pressure disturbances in the axial $(X)$ and off-track $(Y)$ directions. Note that the $X$ and $Y$ coordinates have been transformed to a rectangular space for ease of visualization. For both concepts, the near-field solution is computed for a maximum azimuth angle of $45 \mathrm{deg}$, which produces a maximum off-track location of $63,809 \mathrm{ft}$. 


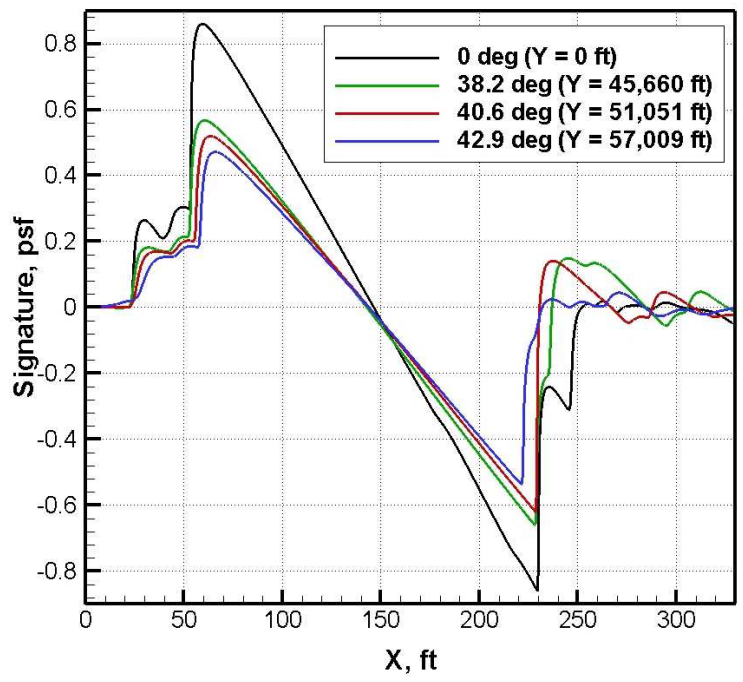

Figure 11. sBOOM signatures for ring wing concept at under- and off-track locations.

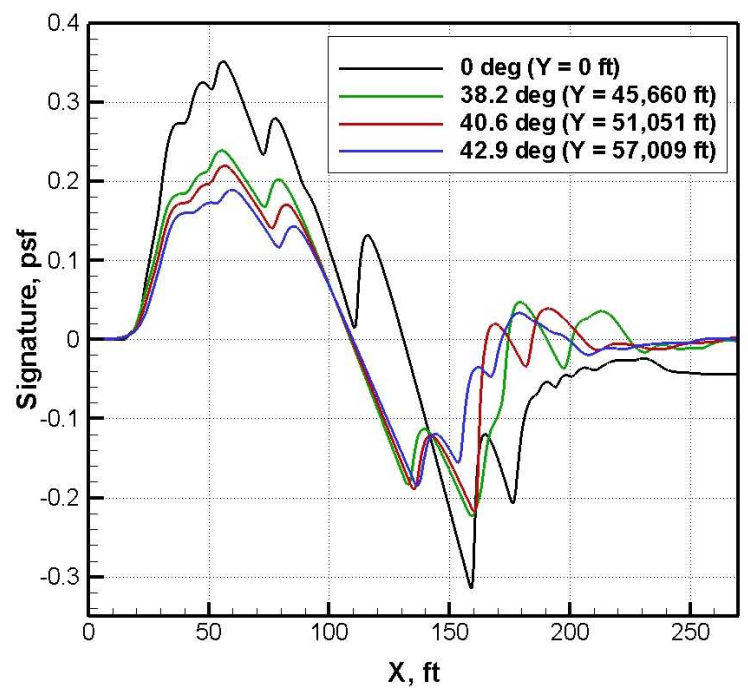

Figure 12. sBOOM signatures for low-boom demonstrator at under- and off-track locations.

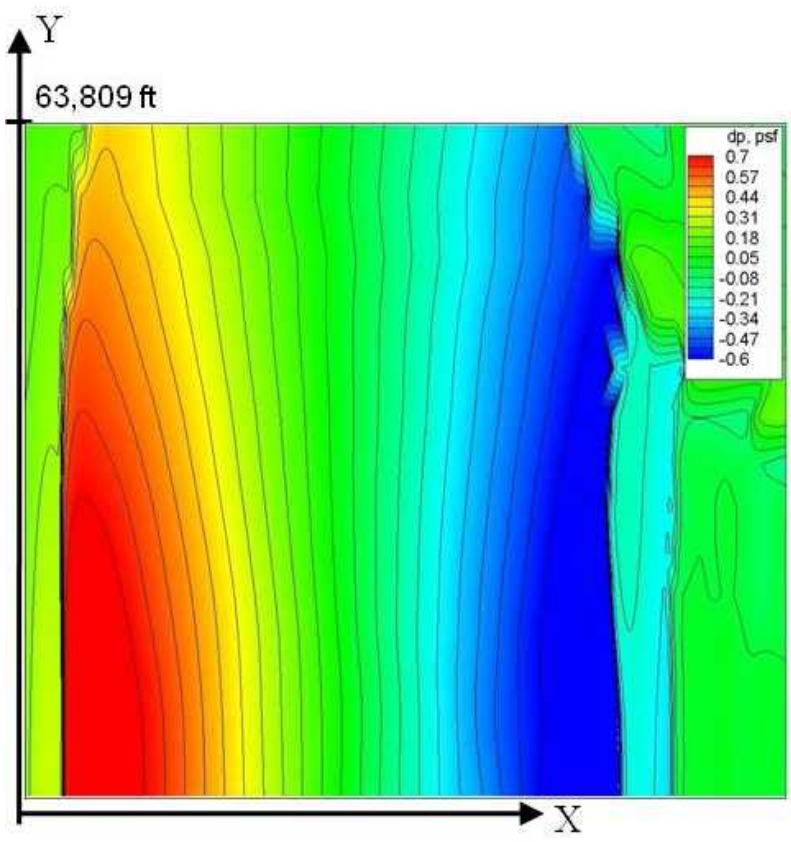

(a) Ring wing.

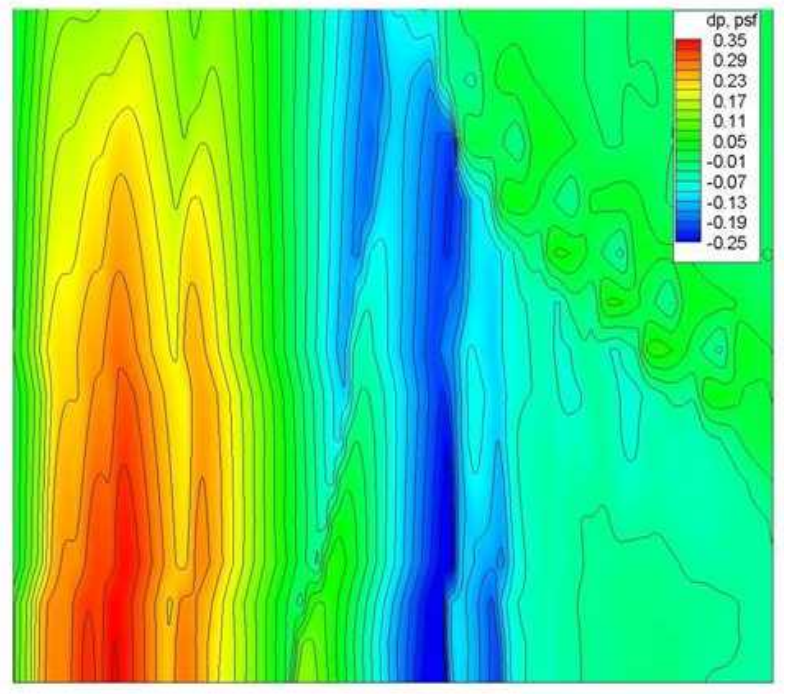

(b) Low-boom demonstrator:

Figure 13. Sonic boom carpets.

The forward, backward, and total loudness levels at each azimuth location for both concepts are shown in Figs. 14 and 15. The low-boom demonstrator displays, as expected, lower loudness levels than the nonoptimized ring wing concept for both the under- and off-track regions. However, the coalescing of the shocks in the off-track direction for the low-boom demonstrator was much more pronounced than for the ring wing concept. Despite this, after the shocks coalesce, the off-track loudness continues to dissipate much more rapidly for the low-boom demonstrator, with a total loudness drop of approximately 12.8 PLdB (15 percent) at 63,809 ft. In contrast, the ring wing loudness levels only decrease 6.9 PLdB (7.2 percent).

The forward and backward loudness contributions were plotted to understand the cause of the loudness rise at an azimuth angle of $40.6 \mathrm{deg}(51,051 \mathrm{ft})$ in the off-track direction. In Figs. 14 and 15, one can see that the loudness rise is caused by the aft portion of the signature. The reason for this increase in loudness can be observed in the signature plots (Figs. 11 and 12) at an azimuth angle of $40.6 \mathrm{deg}(\mathrm{Y}=$ 
$51,051 \mathrm{ft}$ ), which is shown by the red curve. Comparing this signature with the previous (green) and the following (blue) signatures, one can observe that the pressure variations have coalesced into a single and larger aft shock. The reason that these appear at the same location for both concepts may simply be due to the fact that only 15 off-track locations were sampled.

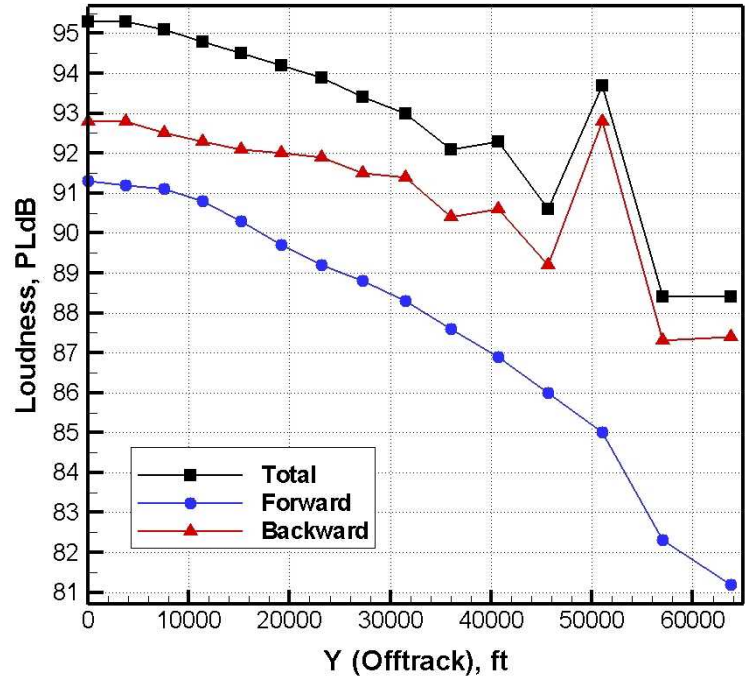

Figure 14. Under- and off-track loudness prediction for ring wing concept.

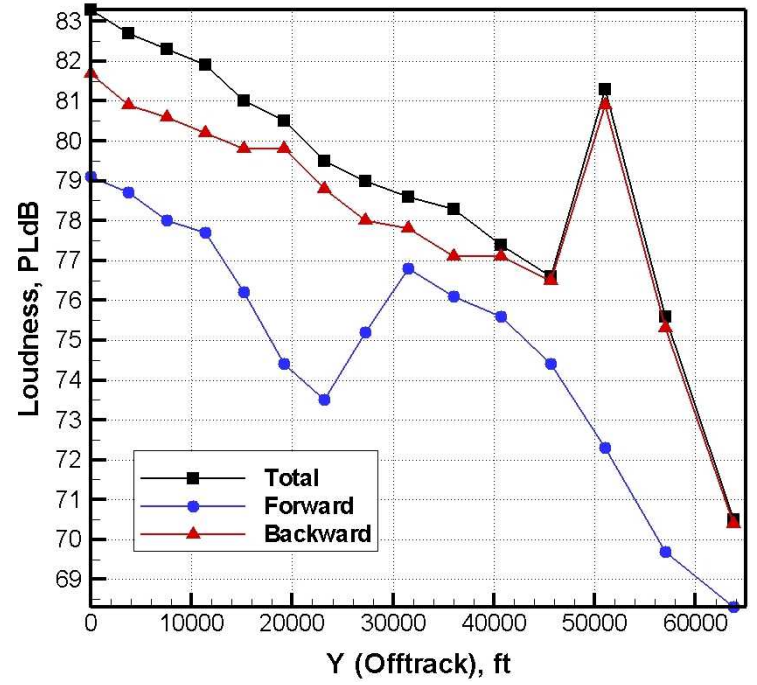

Figure 15. Under- and off-track loudness prediction for low-boom demonstrator.

\section{Concluding Remarks}

A flexible, robust, and automated off-track boom analysis process for the conceptual design of lowboom aircraft has been demonstrated for two challenging supersonic configurations. The ModelCenter model provides a consistent process for obtaining a complete picture of the sonic boom characteristics of complex configurations.

The accuracy of the near-field solutions for Cart3D have been assessed by comparing them with the well-established CFD code, USM3D. Furthermore, the sensitivity of ground signatures to the CFD mesh refinement and to the initial location of the near-field waveform has been successfully documented for the low-boom demonstrator. Lastly, sonic boom carpet analysis results have been presented for both the ring wing concept and the low-boom demonstrator. Results demonstrate that off-track boom analysis can exhibit highly complex and sometimes unexpected behaviors. Furthermore, low-boom designs can potentially exhibit stronger shock coalescence despite having better overall boom characteristics.

This work can be taken a step further to map the sonic boom carpets over terrain and population data. Variations in the type of terrain and in elevation will further affect the characteristics of the ground signatures. Population densities can be used to quantify the environmental impact and facilitate the planning of future flight trajectories.

\section{References}

${ }^{1}$ Howe, D. C., Waithe, K. A., and Haering, E. A., "Quiet Spike Near Field Flight Test Pressure Measurements with Computational Fluid Dynamics Comparisons," AIAA-2008-128, 2008.

${ }^{2}$ Campbell, R., Carter, M., Deere, K., and Waithe, K., "Efficient Unstructured Grid Adaptation Methods for Sonic Boom Prediction," AIAA-2008-7327, August 2008.

${ }^{3}$ Wintzer, M., Nemec, M., and Aftosmis, M., "Adjoint-Based Adaptive Mesh Refinement for Sonic Boom Prediction," AIAA-2008-6593, August 2008.

${ }^{4} \mathrm{Li}, \mathrm{W}$. , Campbell, R., Geiselhart, K., Shields, E., Nayani, S., and Shenoy, R., "Integration of Engine, Plume, and CFD Analyses in Conceptual Design of Low-Boom Supersonic Aircraft," AIAA-2009-1171, January 2009.

${ }^{5} \mathrm{Li}$, W., Shields, E., and Geiselhart, K., "A Mixed-Fidelity Approach for Design of Low-Boom Supersonic Aircraft," AIAA-2010-845, January 2010.

${ }^{6}$ Frink, N., Pirzadeh, S., Parikh, P., Pandya, M., and Bhat, M., "The NASA Tetrahedral Unstructured Software System," The Aeronautical Journal, Vol. 104, No. 1040, 2000, pp. 491-499.

7 "USM3D Users Manual Version 6.0," http://tetruss.larc.nasa.gov/usm3d/, [cited August 18, 2009].

${ }^{8}$ Aftosmis, M., "Cart3D Resource Website," http://people.nas.nasa.gov/ aftosmis/cart3d/cart3Dhome.html, [cited December 2009]. 
${ }^{9}$ Pirzadeh, S., "Three-Dimensional Unstructured Viscous Grids by the Advancing-Layers Method," AIAA Journal, Vol. 34, No. 1, 1996, pp. 43-49.

10 "VGRID Unstructured Grid Generator," http://tetruss.larc.nasa.gov/vgrid/, [cited August 18, 2009].

${ }^{11}$ Hann, A., "Vehicle Sketch Pad: A Parametric Geometry Modeler for Conceptual Aircraft Design," AIAA-2010-657, January 2010.

${ }^{12}$ Ordaz, I. and Rallabhandi, S. K., "Boom Minimization Framework for Supersonic Aircraft Using CFD Analysis," AIAA-2010-1506, January 2010.

${ }^{13}$ Rallabhandi, S. K., "Advanced Sonic Boom Prediction Using Augmented Burger's Equation," AIAA No. Pending, January 2011.

14 "ModelCenter and Optimization Tools," Phoenix Integration, http://www.phoenix-int.com/, [cited December 2009].

${ }^{15}$ Gloudemans, J. R. and McDonald, R., "Improved Geometry Modeling for High Fidelity Parametric Design," AIAA2010-659, January 2010.

${ }^{16}$ Ordaz, I., "Conversion of Component-Based Point Definition to VSP Model and Higher Order Meshing," AIAA No. Pending, January 2011.

${ }^{17} \mathrm{Li}$, W. and Shields, E., "Numerical Optimization of Parametric Equivalent Area Distribution for Design of Low-Boom Supersonic Concepts," AIAA No. Pending, January 2011.

${ }^{18}$ Geiselhart, K. A., Ozoroski, L. P., Fenbert, J. W., Shields, E. W., and Li, W., "Integration of Multifidelity Multidisciplinary Computer Codes for Design and Analysis of Supersonic Aircraft," AIAA No. Pending, January 2011.

${ }^{19}$ Campbell, L., Carter, M., Deere, K., and Waithe, K., "Efficient Unstructured Grid Adaptation Methods for Sonic Boom Prediction," AIAA-2008-7327, August 2008. 